\title{
Anomalous Coronary Arteries: A Case of Rare and Incidental Findings
}

\author{
Mihir Odak $^{\mathrm{a}, \mathrm{c}}$, Ndausung Udongwo ${ }^{\mathrm{a}}$, Dhaval Desai ${ }^{\mathrm{b}}$, Matthew Schoenfeld ${ }^{\mathrm{b}}$
}

\begin{abstract}
Anomalous coronary artery from the opposite sinus (ACAOS) is an uncommon, yet highly variable anatomical abnormality. These coronary anatomy variants are often discovered incidentally during cardiac catheterization. These variants can be challenging intraoperatively and require adjustment by the operator. We present the case of a 93-year-old female who presented for shortness of breath due to severe mitral regurgitation (MR), who was found to have an anomalous left main coronary artery (LMCA) from the right sinus of Valsalva (RSOV). This asymptomatic finding was managed conservatively and patient underwent successful MitraClip treatment.
\end{abstract}

Keywords: Coronary artery anomaly; Congenital heart disease; Right coronary cusp; Coronary sinus

\section{Introduction}

Anomalous coronary artery from the opposite sinus (ACAOS) is a rare and varied phenomenon. While there are reports of anomalous coronary arteries in literature, the frequency of each anomaly is highly variable and these findings are almost always incidental. Early reporting of anomalous coronary arteries dated as early as 1948, when Whites and Edwards described an anomalous right coronary artery (RCA) [1]. In years since then, as interventions and diagnostic technologies have advanced, an increase in the frequency of anomalous coronary arteries has been observed. We present a case of a 93-year-old female who was admitted for acute diastolic heart failure exacerbation secondary to mitral valve prolapse with severe mitral regurgitation. An evaluation for MitralClip ther-

Manuscript submitted September 23, 2021, accepted October 20, 2021

Published online November 5, 2021

aDepartment of Internal Medicine, Jersey Shore University Medical Center, Neptune City, NJ 07753, USA

${ }^{b}$ Department of Cardiology, Jersey Shore University Medical Center, Neptune City, NJ 07753, USA

${ }^{\mathrm{c}}$ Corresponding Author: Mihir Odak, Department of Internal Medicine, Jersey Shore University Medical Center, 1945 NJ-33, Neptune City, NJ 07753, USA. Email:mihir.odak@hmhn.org

doi: https://doi.org/10.14740/jmc3782 apy revealed an anomalous left main coronary artery (LMCA) arising from the right sinus of Valsalva (RSOV) during cardiac catheterization.

\section{Case Report}

\section{Investigations}

The patient is a 93-year-old female who presented to the emergency department (ED) with worsening shortness of breath that started approximately 6 days prior to admission. She has a past medical history of mitral valve prolapse with moderate mitral regurgitation, heart failure with preserved ejection fraction $(\mathrm{HFpEF})$ with an ejection fraction (EF) of $65-70 \%$, atrial fibrillation on anticoagulation, hypertension, and macular degeneration. Her symptoms occurred with minimal exertion and were associated with palpitations, fatigue, decreased appetite, and lower extremity edema. She denied any chest pain. Her home medications were furosemide $40 \mathrm{mg}$ twice daily, potassium chloride $10 \mathrm{mg}$ daily, hydralazine $50 \mathrm{mg}$ daily, losartan $100 \mathrm{mg}$ daily, apixaban $2.5 \mathrm{mg}$ every $12 \mathrm{~h}$, and amlodipine 10 mg daily.

On admission, her blood pressure was 211/87 $\mathrm{mm} \mathrm{Hg}$, heart rate 84 beats per minute, oxygen saturation $89 \%$ on room air, and she was afebrile. She was in no acute distress with a 3/6 holosystolic murmur at the apex, elevated jugular venous pressure, bibasilar rales, and $2+$ bilateral lower extremity edema. Her oxygen saturation increased to $99 \%$ on $4 \mathrm{~L}$ nasal cannula.

\section{Diagnosis}

Upon admission, laboratory studies were obtained, which are detailed in Table 1.

An electrocardiogram was also obtained, which revealed atrial fibrillation, with a rate of 75 beats per minute, right axis deviation, with non-specific ST-T waves changes (Fig. 1). Chest X-ray revealed cardiomegaly with bilateral pulmonary infiltrates (Fig. 2).

A transthoracic echocardiogram revealed normal left ventricle (LV) size with left ventricle ejection fraction (LVEF) of $65-70 \%$ with bileaflet mitral valve prolapse and severe mitral regurgitation. A transesophageal echocardiogram (TEE) was performed for further evaluation of her mitral regurgitation which confirmed bileaflet mitral valve prolapse with a later- 
Table 1. Laboratory Studies Upon Admission

\begin{tabular}{lll}
\hline Laboratory & Results & References \\
\hline Hemoglobin $(\mathrm{g} / \mathrm{dL})$ & 11.5 & $12.0-16$ \\
White blood cell count $\left(\times 10^{9} / \mathrm{L}\right)$ & 10.2 & $4.5-11.0$ \\
Sodium $(\mathrm{mmol} / \mathrm{L})$ & 134 & $135-146$ \\
Potassium $(\mathrm{mmol} / \mathrm{L})$ & 5.5 & $3.5-5.0$ \\
Glucose $(\mathrm{mg} / \mathrm{dL})$ & 134 & $70-110$ \\
Blood urea nitrogen $(\mathrm{mg} / \mathrm{dL})$ & 37 & $7.0-18.0$ \\
Creatinine $(\mathrm{mg} / \mathrm{dL})$ & 1.15 & $0.44-1.0$ \\
B-type natriuretic peptide $(\mathrm{pg} / \mathrm{mL})$ & 719 & $0-100$ \\
Aspartate aminotransferase $(\mathrm{U} / \mathrm{L})$ & 62 & $10-42$ \\
Alanine aminotransferase $(\mathrm{U} / \mathrm{L})$ & 30 & $10-60$ \\
Total bilirubin $(\mathrm{mg} / \mathrm{dL})$ & 1.8 & $0.2-1.2$ \\
\hline
\end{tabular}

al jet and severe mitral regurgitation (estimated orifice area $0.4 \mathrm{~cm}^{2}$ ). Given her age she was recommended for MitraClip evaluation. A pre-MitraClip left heart catheterization was performed, during which we were unable to cannulate the coronary arteries from the radial approach due to severe subclavian and brachiocephalic artery tortuosity. A femoral approach was therefore favored.

\section{Treatment}

The patient was started on intravenous furosemide $40 \mathrm{mg}$ and nitroglycerin infusion with improvement in her blood pressure and oxygen saturation. Catheterization via the femoral approach revealed an anomalous left main coronary artery (LMCA) arising from the right coronary cusp (Fig. 3a-c), which was subsequently successfully cannulated with a 6-Fr

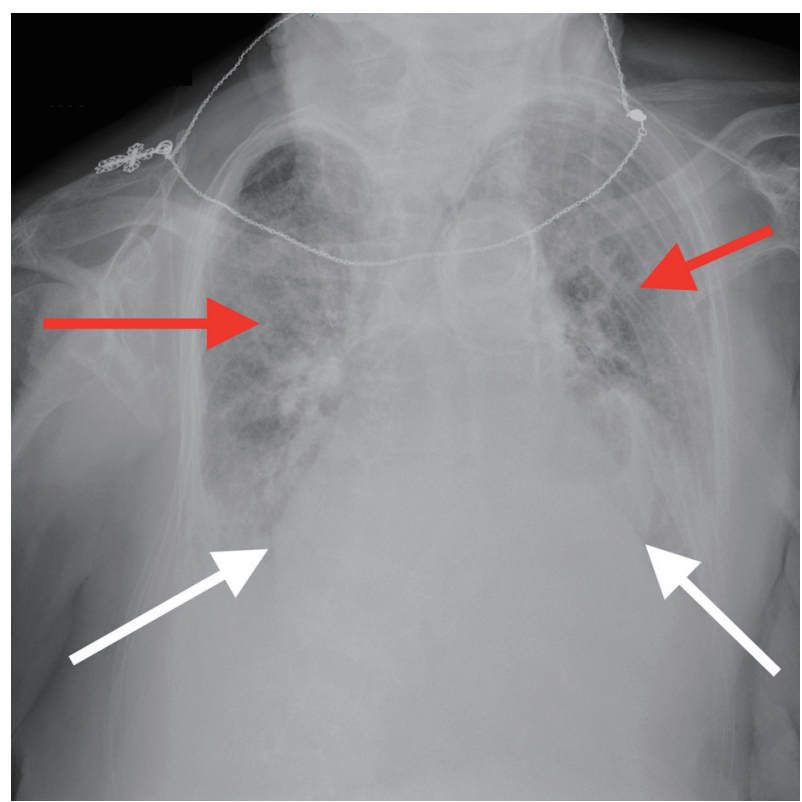

Figure 2. Chest $\mathrm{X}$-ray showing cardiomegaly (white arrows showing the cardiac borders) and extensive bilateral pulmonary vascular congestion (red arrows).

JR4.0 diagnostic catheter. Both arteries were angiographically free of atherosclerotic disease. She subsequently underwent successful MitraClip procedure with one clip.

\section{Follow-up and outcomes}

Following her MitraClip procedure, the patient was found to have only mild residual mitral regurgitation and significant improvement in her symptoms.

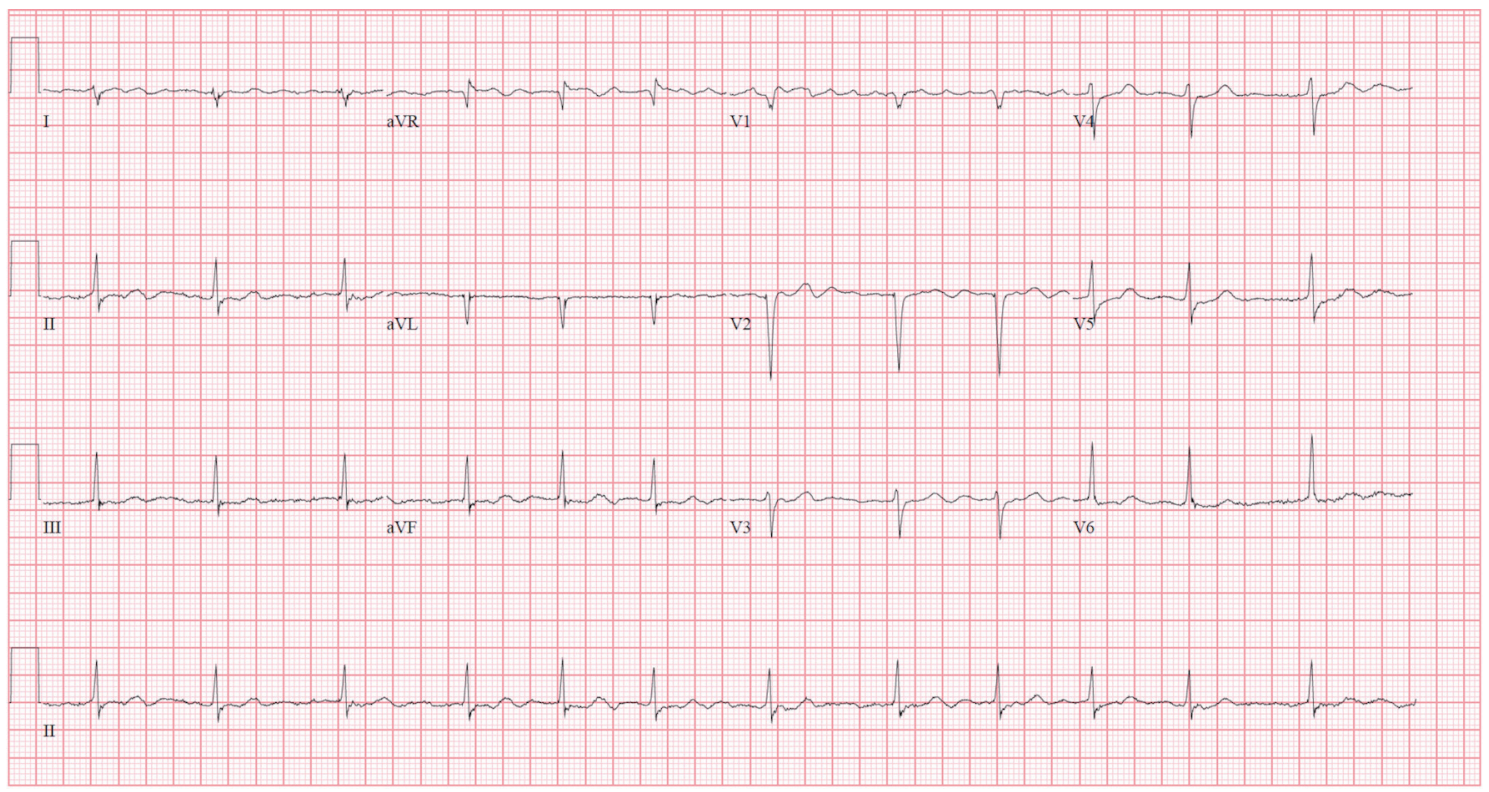

Figure 1. Presenting electrocardiogram showing atrial fibrillation with rate to 75 beats per minute, without ST-T changes. 


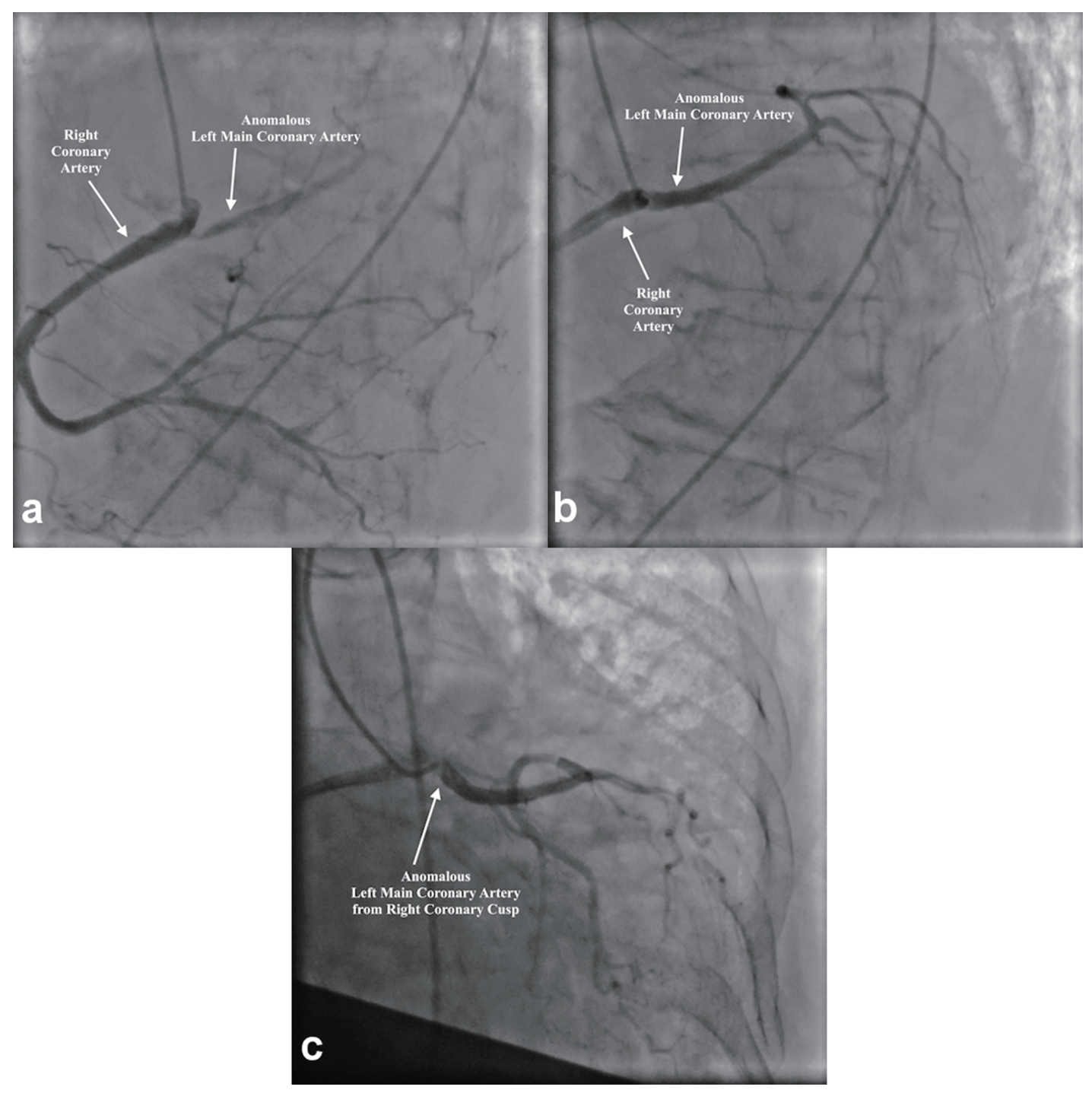

Figure 3. (a) Cannulation and angiography of the RCA (white arrows). (b, c) Simultaneous angiography of the LMCA and RCA due to anomalous origin of LMCA from RSOV (white arrows). RCA: right coronary artery; LMCA: left main coronary artery; RSOV: right sinus of Valsalva.

\section{Discussion}

Coronary artery anomalies (CAAs) are rare and found in approximately $1 \%$ of the population $(0.3-5.6 \%)$ [2]. They are discovered in approximately 13 per 1,000 people undergoing cardiac catheterization [3]. The most common CAAs include separate ostia of the left anterior descending artery (LAD) and left circumflex artery (LCX) followed by anomalous LCX from the RCA. Here we present a case of the more uncommon LMCA from the RSOV, which has a reported incidence of $0.0024-0.02 \%$ [3].

CAAs are most commonly asymptomatic and diagnosed incidentally [4]. Symptomatic presentations are rare but include chest pain, syncope, and sudden cardiac death $[4,5]$. The risk of ischemia is highly dependent on the course of the anomaly with an inter-arterial course with slit-like ostia having the highest risk [5]. Once diagnosed, these patients should un- dergo coronary computed tomography angiography (CTA) to evaluate for high risk anatomy, as well as functional ischemic evaluation with stress testing. LMCA from RCA is particularly high risk for sudden cardiac death in cases of inter-arterial courses with intramural involvement, slit-like ostia, and acute angle take-offs of the LMCA. Such cases should carry a low threshold for surgery, especially if patients present with symptoms suggestive of acute coronary syndrome [6]. Asymptomatic patients, who have pre-pulmonic courses, are not referred for surgery as such courses are benign and the reported incidence of ischemia in these individuals is low [7]. Our patient's absence of coronary disease was further supportive of a medical management strategy. Such patients can be reassured and managed conservatively [6].

CAAs can cause challenges in engaging coronary arteries which can lead to delays in acute coronary syndrome settings. For ACAOS with LMCA from the RSOV, the course of the 
LMCA is best visualized in the right anterior oblique (RAO) caudal view, and its course may run inter-arterial, retro-aortic, septal, or anterior-pulmonic [8]. Judkins Right 4 (JR4) and hockey stick catheters can be used in these scenarios to engage the RCA and with rotation, the LMCA [9].

\section{Learning points}

Anomalous coronary arteries have increased in incidence with the development of imaging. Certain variants of coronary anatomy, such as ACAOS with LMCA from RCA can be significantly high-risk depending on the course. In older patients, especially those without ischemic symptoms, this incidental finding is benign and patients should be reassured.

\section{Acknowledgments}

The authors have no further acknowledgements to disclose.

\section{Financial Disclosure}

None to declare.

\section{Conflict of Interest}

None to declare.

\section{Informed Consent}

Informed consent was obtained from the patient prior to presentation of this manuscript.

\section{Author Contributions}

Mihir Odak: preparation of manuscript; Ndausung Udongwo: preparation of manuscript; Dhaval Desai: preparation of man- uscript; Matthew Schoenfeld: senior author, procedure operator, preparation and editing of manuscript.

\section{Data Availability}

The authors declare that data supporting the findings of this study are available within the article.

\section{References}

1. Keswani AN, Dann K, Ramee S. Anomalous right coronary artery: a case report. Ochsner J. 2014;14(2):287291.

2. Villa AD, Sammut E, Nair A, Rajani R, Bonamini R, Chiribiri A. Coronary artery anomalies overview: The normal and the abnormal. World J Radiol. 2016;8(6):537555.

3. Kilic A, Kilic A, Sultan I. Anomalous origin of the left main coronary artery from the right coronary artery. Circ Cardiovasc Imaging. 2018;11(12):e008452.

4. Grani C, Kaufmann PA, Windecker S, Buechel RR. Diagnosis and management of anomalous coronary arteries with a malignant course. Interv Cardiol. 2019;14(2):8388.

5. Suryanarayana P, Lee JZ, Abidov A, Lotun K. Anomalous right coronary artery: case series and review of literature. Cardiovasc Revasc Med. 2015;16(6):362-366.

6. Rinaldi RG, Carballido J, Giles R, Del Toro E, Porro R. Right coronary artery with anomalous origin and slit ostium. Ann Thorac Surg. 1994;58(3):829-832.

7. Brothers JA, Frommelt MA, Jaquiss RDB, Myerburg RJ, Fraser CD, Jr., Tweddell JS. Expert consensus guidelines: Anomalous aortic origin of a coronary artery. J Thorac Cardiovasc Surg. 2017;153(6):1440-1457.

8. Angelini P. Coronary artery anomalies: an entity in search of an identity. Circulation. 2007;115(10):1296-1305.

9. Sinha SK, Razi M, Mahrotra A, Aggarwal P, Singh A, Rekwal L, Tripathi S, et al. Anomalous coronary artery from the opposite sinus (ACAOS): technical challenges during percutaneous coronary intervention. Cardiol Res. 2018;9(2):120-124. 